\title{
Combinatorics of 4-dimensional Resultant Polytopes
}

\author{
Alicia Dickenstein ${ }^{*}$ \\ Dto. de Matemática, FCEN \\ Universidad de Buenos Aires, \\ \& IMAS-CONICET, Argentina \\ alidick@dm.uba.ar
}

\author{
loannis Z. Emiris ${ }^{*}$ \\ Department of Informatics \\ and Telecommunications \\ University of Athens, Greece \\ emiris@di.uoa.gr
}

\author{
Vissarion Fisikopoulos \\ Department of Informatics \\ and Telecommunications \\ University of Athens, Greece \\ vissarion@di.uoa.gr
}

\begin{abstract}
The Newton polytope of the resultant, or resultant polytope, characterizes the resultant polynomial more precisely than total degree. The combinatorics of resultant polytopes are known in the Sylvester case [4] and up to dimension 3 [9]. We extend this work by studying the combinatorial characterization of 4-dimensional resultant polytopes, which show a greater diversity and involve computational and combinatorial challenges. In particular, our experiments, based on software respol for computing resultant polytopes, establish lower bounds on the maximal number of faces. By studying mixed subdivisions, we obtain tight upper bounds on the maximal number of facets and ridges, thus arriving at the following maximal $f$-vector: $(22,66,66,22)$, i.e. vector of face cardinalities. Certain general features emerge, such as the symmetry of the maximal $f$-vector, which are intriguing but still under investigation. We establish a result of independent interest, namely that the $f$-vector is maximized when the input supports are sufficiently generic, namely full dimensional and without parallel edges. Lastly, we offer a classification result of all possible 4-dimensional resultant polytopes.
\end{abstract}

\section{Categories and Subject Descriptors}

G.2.1 [Discrete Mathematics]: Combinatorics-Counting problems; I.1.2 [Symbolic and Algebraic Manipulation]: Algorithms-Algebraic algorithms

\section{Keywords}

Resultant, $f$-vector, Mixed subdivision, Secondary polytope

\section{INTRODUCTION}

\footnotetext{
*Partially supported by UBACYT 20020100100242, CONICET PIP 112-200801-00483 and ANPCyT PICT 2008-0902, Argentina. ** Partially supported by "Computational Geometric Learning", financed within the 7th Framework Program for research of the European Commission, under FET-Open grant number 255827.
}

Permission to make digital or hard copies of all or part of this work for personal or classroom use is granted without fee provided that copies are not made or distributed for profit or commercial advantage and that copies bear this notice and the full citation on the first page. To copy otherwise, to republish, to post on servers or to redistribute to lists, requires prior specific permission and/or a fee.

ISSAC'13, June 26-29, 2013, Boston, Massachusetts, USA.

Copyright 2013 ACM 978-1-4503-2059-7/13/06 ...\$10.00.
Let $\mathcal{A}=\left(A_{0}, \ldots, A_{n}\right)$ be a family of subsets of $\mathbb{Z}^{n}$ and let $f_{0}, \ldots, f_{n} \in \mathbb{C}\left[x_{1}, \ldots, x_{n}\right]$ be polynomials with this family of sets as supports, and symbolic coefficients $c_{i j} \neq 0$, $i=0, \ldots, n, j=1, \ldots,\left|A_{i}\right|$, i.e. $f_{i}=\sum_{a \in A_{i}} c_{i j} x^{a}$. The family $\mathcal{A}$ is essential if these sets jointly affinely span $\mathbb{Z}^{n}$, and every subfamily of $A_{i}$ 's of cardinality $j, 1 \leq j<n$, spans an affine space of dimension $\geq j$. In this paper we assume that $\mathcal{A}$ is essential. The sparse (or toric) resultant $\mathcal{R}=\mathcal{R}_{\mathcal{A}}$ of $f_{0}, \ldots, f_{n}$ is then a non-constant irreducible polynomial in $\mathbb{Z}\left[c_{i j}: i=0, \ldots, n, j=1, \ldots,\left|A_{i}\right|\right]$, defined up to sign, which vanishes if $f_{0}=f_{1}=\cdots=f_{n}=0$ has a solution in $\left(\mathbb{C}^{*}\right)^{n}, \mathbb{C}^{*}=\mathbb{C} \backslash\{0\}$. The Newton polytope $N(\mathcal{R})$ of the resultant, that is, the convex hull of the exponents occurring in $\mathcal{R}$ with non-zero coefficient, is a lattice polytope called resultant polytope. A famous example is the Birkhoff polytope of a linear system, cf Example 1.

The resultant has $m=\sum_{i=0}^{n}\left|A_{i}\right|$ variables, hence $N(\mathcal{R})$ lies in $\mathbb{R}^{m}$. However, for essential families, $\mathcal{R}$ satisfies $n+(n+$ 1) natural homogeneities [5], so its dimension is $\operatorname{dim}(N(\mathcal{R}))=$ $m-2 n-1$. If $\mathcal{A}$ is not essential, but contains a single essential subfamily, the resultant depends only on the coefficients of the polynomials in this subfamily. Otherwise, the resultant locus has codimension bigger than one, and then the sparse resultant is defined to be the constant 1 .

Previous work. In [3] an algorithm is described for computing the vertex and facet representations of $N(\mathcal{R})$; the algorithm also produces a triangulation of the polytope's interior into simplices. The input to this algorithm is an oracle for computing the extreme resultant vertex given a direction. The software implementation is called respol ${ }^{1}$ and is the one used in our experiments. This method is readily generalized to compute the discriminant and secondary polytopes, although for the latter there exists a faster method to enumerate the vertices, when only these are needed [8]. An alternative way for computing resultant polytopes exploits tropical geometry [6] and is implemented based on the software library Gfan.

The combinatorics of resultant polytopes is known only in small cases, namely for linear systems (Example 1), in the Sylvester case $(n=1)$, and when $\operatorname{dim} N(\mathcal{R})=3$. The univariate case is fully described in [4]: $N(\mathcal{R})$ is combinatorially isomorphic to a polytope denoted by $\mathcal{N}_{k_{0}, k_{1}}$, of dimension $k_{0}+k_{1}-1$, where the $A_{i}$ may be multisets with cardinality $k_{i}$. They may lead to polytopes in any dimension if one picks the $A_{i}$ accordingly. In [5] they show that $\mathcal{N}_{k_{0}, k_{1}}$ has $\left(\begin{array}{c}k_{0}+k_{1} \\ k_{0}\end{array}\right)$ vertices and, when both $k_{i} \geq 2$, it has $k_{0} k_{1}+3$

$\overline{{ }^{1} \text { http://respol.sourceforge.net }}$ 
facets. Sturmfels [9] classifies all resultant polytopes up to dimension 3. In his notation, the 3-dimensional polytope $\mathcal{N}_{1111,111}$, denoted by $\mathcal{N}_{3,2}$ in [4], depicted in Fig. 1 with the resultant label, has maximal $f$-vector.

Proposition 1. [9, Sect. 6] Assume $\mathcal{A}$ is an essential family. Then, $N(\mathcal{R})$ is 1-dimensional iff $\left|A_{i}\right|=2$, for all $i$. The only planar resultant polytope is the triangle. The only 3-dimensional $N(\mathcal{R})$ are, combinatorially: (a) the tetrahedron, (b) the square-based pyramid, and (c) the polytope $\mathcal{N}_{3,2}$.

In [6] the authors raise explicitly the open question of describing 4-dimensional resultant polytopes, which we undertake in this paper.

Our contribution. We study the combinatorial characterization of 4-dimensional resultant polytopes. To bound the maximum number of faces, we prove that it suffices to focus on one case, which corresponds to 3 Newton polygons with support cardinalities $\left|A_{i}\right|=3$, thus $m=9$ and $n=2$. We further show it is enough to consider sufficiently generic polygons, namely where they all have non-zero area, and no parallel edges exist among them. Our experiments, based on respol [3], establish lower bounds on the maximal number of faces (Table 1). By studying mixed subdivisions, we obtain tight upper bounds on the maximal number of facets and ridges, thus raising new conjectures, the most important of which is that the maximal $f$-vector is $(22,66,66,22)$ for 4-dimensional $N(\mathcal{R})$. These results are summarized in Thm 16. Our (loose) upper bound on the number of vertices, namely 28, significantly improves the known bound of 6608 [9, Cor.6.2]. Certain general features emerge, such as the symmetry of the maximal $f$-vector, which are intriguing but still under investigation. However, the Newton polytopes are not self-dual. Our main result is Thm 17, where we offer a characterization of all possible 4-dimensional resultant polytopes.

The rest of the paper is organized as follows. The next section introduces 4-dimensional resultant polytopes. Sect. 3 focuses on three $2 \mathrm{~d}$-triangles with non-parallel edges, which maximizes the number of faces, and upper bounds the number of facets and ridges in $N(\mathcal{R})$ by combinatorial arguments. Sect. 4 classifies all 4 -dimensional resultant polytopes, and proves we can ignore parallel edges when maximizing the number of faces. We conclude with open questions and generalizations.

\section{RESULTANT POLYTOPES}

We start with previous results from $[9,5]$. The main tool for computing sparse resultants are the regular mixed subdivisions of the convex hull of Minkowski sum $P=\sum_{i} A_{i}$. By abuse of notation, we may also refer to this sum as $\sum_{i} P_{i}$, where $P_{i}$ denotes the convex hull of $A_{i}$ and it is understood that the information from the $A_{i}$ 's is preserved. A subdivision of $P$ is a collection of subsets of $P$, the cells of the subdivision, such that the union of the cells' convex hulls equals the convex hull of $P$ and every pair of convex hulls of cells intersect at a common face. Maximal cells are those with dimension equal to the dimension of the subdivision. Fine (or tight) are those whose dimension equals the sum of its summands' dimensions. A subdivision is regular if it can be obtained as the projection of the lower hull of the Minkowski sum $\sum_{i} \widehat{A}_{i}$ of the lifted point sets $\widehat{A}_{i}$, for some lifting to $\mathbb{R}^{n+1}$. A subdivision is mixed when its cells have (unique) expressions as Minkowski sums of convex hulls of point subsets in the $A_{i}$ 's. It is fine (or tight) if all its cells are fine. Maximal cells are mixed if the dimension of every summand, except possibly one, equals one. In the sequel, we focus only on regular subdivisions, thus we will omit the word regular in general.

Given a family $\mathcal{A}$, the associated Cayley configuration $\mathcal{C}$ is the lattice configuration in $\mathbb{Z}^{n+1} \times \mathbb{Z}^{n}=\mathbb{Z}^{2 n+1}$ defined by

$$
\left\{e_{0}\right\} \times A_{0} \cup \cdots \cup\left\{e_{n}\right\} \times A_{n},
$$

where $e_{0}, \ldots, e_{n}$ denotes the canonical basis in $\mathbb{Z}^{n+1}$. We denote by $Q$ its convex hull. Regular fine mixed subdivisions of $P$ are in bijection with regular triangulations of $Q$. Indeed, there is a bijection of maximal cells given as follows: any maximal cell (simplex) $\sigma_{T}$ in a given regular triangulation $T=T_{w}$ of $Q$ (with vertices in $\mathcal{C}$ ) has $2 n$ vertices; the corresponding maximal cell in the associated regular fine subdivision $S=S_{w}$ of $P$ has vertices of the form $\alpha_{0}+\cdots+\alpha_{n}$, with $\left(e_{i}, \alpha_{i}\right)$ a vertex of $\sigma_{T}$. Note that for $\sigma_{T}$ to be of maximal dimension, at least one of its vertices lies in $e_{i} \times A_{i}$, for all $i$. For more details about the translation between regular subdivisions of $Q$ and regular mixed subdivisions of $P$, see [2, Sect. 9.2].

Let $C$ be the $(2 n+1) \times m$ associated Cayley matrix, i.e., the matrix whose columns are the points in the Cayley configuration $\mathcal{C}$. The inner product of any point in $N(\mathcal{R})$ with any vector in the rowspan of the Cayley matrix $C$ is constant, and so $N(\mathcal{R})$ lies in a parallel translate to the nullspace of $C$. This explains why $\operatorname{dim}(N(\mathcal{R}))=m-2 n-1$.

The faces (resp. vertices) of $N(\mathcal{R})$ can be obtained, by a many-to-one mapping, from the set of all regular (resp. fine) mixed subdivisions of $P$ [9]. Given a mixed subdivision of $P$, every cell $\sigma$ defines a subsystem of the $\left.f_{i}\right|_{\sigma}$, where each polynomial is a restriction of $f_{i}$ on the face of $A_{i}$ appearing as a summand in $\sigma$. If the subdivision is the projection of the lower hull under a lifting $w$, then the face of $N(\mathcal{R})$ whose outer normal is $w$ is

$$
\prod_{\sigma} \mathcal{R}\left(\left.f_{0}\right|_{\sigma}, \ldots,\left.f_{n}\right|_{\sigma}\right)^{d_{\sigma}}
$$

where $d_{\sigma} \in \mathbb{N}$ is specified in [9, Thm 4.1]. We shall call $\sigma$ essential if the corresponding $\left.f_{i}\right|_{\sigma}$ define an essential subsystem. Hence, all faces of $N(\mathcal{R})$ are Minkowski sums of lower-dimensional resultant polytopes, corresponding to essential subsystems. These lower-dimensional resultant polytopes correspond to subsets of the cells of the subdivision defining the face of $N(\mathcal{R})$. In particular, resultant vertices are obtained when all resultants in (1) are monomials, hence all $\sigma$ are mixed; then $d_{\sigma}$ is the normalized volume of $\sigma$.

We call flip the transformation of a fine mixed subdivision of $P$ to another fine mixed subdivision of $P$. Following [9], if these subdivisions correspond to different vertices of $N(\mathcal{R})$ we call this flip cubical. In other words, a cubical flip corresponds to a resultant edge. The above discussion yields the following which, will be our basic tool for counting the faces of $N(\mathcal{R})$.

Proposition 2. [9, Thm.4.1] A mixed subdivision $S$ of $P$ corresponds to a face of $N(\mathcal{R})$, which is the Minkowski sum of the resultant polytopes of the cells of $S$.

\subsection{4-dimensional resultant polytopes.}




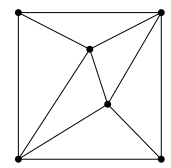

resultant

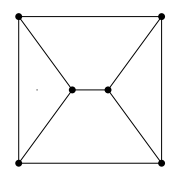

prism

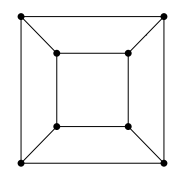

cube
Figure 1: Graph skeleta of $3 \mathrm{~d}$ facets of $4 \mathrm{~d}$ resultant polytopes of 3 triangles with non-parallel edges.

In this case, $m=2 n+5$, where $m=\sum_{i=0}^{n}\left|A_{i}\right|$, and $\left|A_{i}\right| \geq 2$. So, there are only 3 cases, up to reordering:

(i) All $\left|A_{i}\right|=2$, except for one with cardinality 5 .

(ii) All $\left|A_{i}\right|=2$, except for two with cardinalities 3 and 4 .

(iii) All $\left|A_{i}\right|=2$, except for three with cardinality 3 .

Cases (i) and (ii) are similar to the study of 3d-resultant polytopes in [9], cf Thm 17. So, we concentrate on the new case (iii) and, more precisely, on the main case $n=2$ and each $\left|A_{i}\right|=3$, which we term the case $(3,3,3)$. This is done without loss of generality, by the following:

THEOREM 3. [9, Thm.6.2] Every resultant polytope of an essential family is affinely isomorphic to a resultant polytope of an essential family $\left(A_{0}, \ldots, A_{n}\right)$ with $\left|A_{i}\right| \geq 3$, for all $i=0, \ldots, n$.

The proof is an algorithm to produce this reduction: up to an affine change of variables and reordering, we can assume that $A_{i}=\left\{0, \nu_{i} e_{i+1}\right\}, i=0, \ldots, n-3$, so we can solve (with rational powers) the first $n-2$ variables and replace them in the last 3 polynomials. Then, $N(\mathcal{R})$ has the same combinatorial type as an essential $(3,3,3)$ configuration, where we could have repeated points or parallel edges, even if they were not present in $A_{n-2}, A_{n-1}$ and $A_{n}$ (and some coefficients could be equal to the sum of Laurent monomials in the original coefficients).

Let us focus on $N(\mathcal{R})$ for an essential $(3,3,3)$ configuration $\mathcal{A}=\left(A_{0}, A_{1}, A_{2}\right)$, and consider $A_{2}^{\prime} \subset A_{2}$.

Lemma 4. [9] When there are no parallel edges in the subfamily $\left(A_{0}, A_{1}, A_{2}^{\prime}\right),\left|A_{2}^{\prime}\right|=2$, their corresponding resultant polytope gives a facet of $N(\mathcal{R})$, which is combinatorially equivalent to the polytope of Fig 1 (resultant).

\section{THE CASE $(3,3,3)$}

In this section, we assume that we have an essential family with $n=3, m=9$ and each $A_{i}$ has cardinality 3 . Our study shows the richness of possible polytopes, in contrast to the case of resultant polytopes with dimension $\leq 3$.

We write the $f$-vectors as $\left(f_{0}, f_{1}, f_{2}, f_{3}\right)$, omitting the 1 corresponding to the unique 4 -face. We define the minimum and maximum $f$-vector to be the one with minimum and maximum number of facets, i.e. with minimum or maximum value of $f_{3}$. The minimum $f$-vector of the $(3,3,3)$ case when all $P_{i}$ have dimension 2 but we admit parallel edges, is $(6,15,18,9)$ and is attained by the following example.

Example 1 (Birkhoff polytope). Let $A_{0}=A_{1}=$ $A_{2}=\{(0,0),(1,0),(0,1)\}$. Then $N(\mathcal{R})$ is the 4-dimensional Birkhoff polytope [10] which has $f$-vector $(6,15,18,9)$.
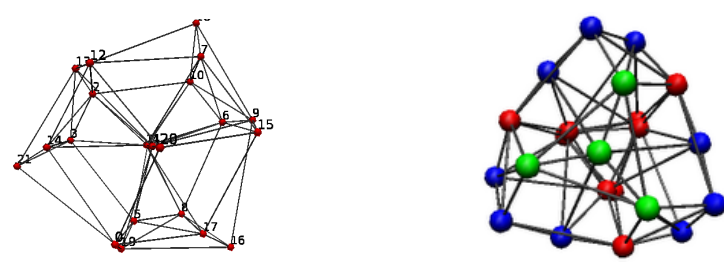

Figure 2: Vertex graph and facet graph (courtesy of M. Joswig) of the resultant polytope in Example 2.

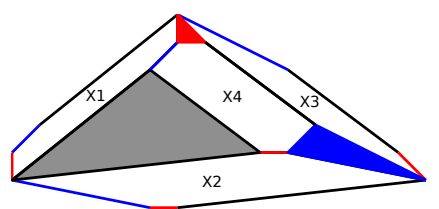

Figure 3: A non-regular subdivision of $P$ of Example 2. There are 4 hexagons X1, X2, X3, X4.

A complete list of $f$-vectors when $A_{0}=\{(0,0),(0,1),(1,0)\}$ and $A_{1}, A_{2}$ take all the possible values from the set $\left\{(0,0), a_{1}\right.$, $\left.a_{2}\right\}$ where $a_{1}, a_{2} \in\{(j, k) \mid j, k \in \mathbb{N} \wedge j, k \leq 5\}$ is presented in Table 1 . There is a unique $f$-vector, $(22,66,66,22)$, which is maximal, and corresponds to more than one input family of supports. Highlighted $f$-vectors correspond to triangles that share no parallel edges between any of them. The computations have been performed using respol and last several days.

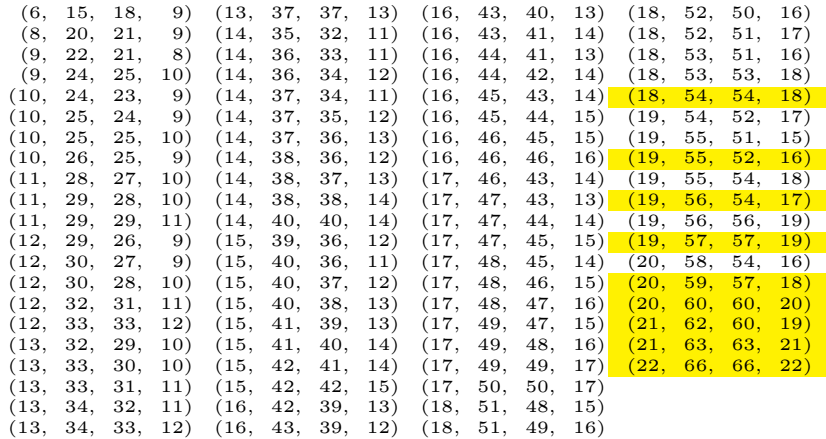

Table 1: The largest $f$-vectors of $4 \mathbf{d} N(\mathcal{R})$ computed: 9 highlighted $f$-vectors correspond to triangles without parallel edges.

A particular extremal case follows:

Example 2. Let $A_{0}=\{(0,0),(1,0),(0,1)\}, A_{1}=\{(0,0)$, $(5,4),(9,1)\}, A_{2}=\{(5,0),(0,1),(1,2)\}$. Then, $N(\mathcal{R})$ has $f$-vector (22,66,66,22); the vertex and facet graphs are in Fig. 2. A non-regular subdivision of $P=\sum_{i} P_{i}$ is depicted in Fig. 3 (see also Rem. 1).

On the other hand, the following example concerns the case of three 1-dimensional configurations, excluded from the above list.

Example 3. Let $A_{0}=\{(0,0),(0,1),(0,2)\}, A_{1}=\{(0,0)$, $(1,0),(2,0)\}, A_{2}=\{(0,0),(1,1),(2,2)\}$. Then, $N(\mathcal{R})$ has $f$-vector $(20,57,51,14)$. 
If we replace the configuration $\left(A_{0}, A_{1}, A_{2}\right)$ in Example 3 by the configuration $\left(A_{0}, A_{1},\{(0,0),(1,1),(2,3)\}\right)$, where two parallelisms are broken without introducing any new one, the $f$-vector becomes $(20,58,54,16)$. Note that we get higher values for the different $f_{i}$.

\subsection{Non-parallel edges}

All along this section, we consider essential $(3,3,3)$ families with $\operatorname{dim}\left(P_{i}\right)=2$, for all $i$, where moreover no edges coming from two different $P_{i}$ are parallel. Even in this case, there are several different relevant polytopes (cf highlighted $f$-vectors in Table 1 ).

We describe the subsets $A_{i}^{\prime} \subset A_{i}$, which form subsystems, that define cells of a subdivision $S$ of $P$, and their connection to the faces of $N(\mathcal{R})$. The simpler non-trivial subsystem is 2-element subsets $A_{i}^{\prime}$ in each $A_{i}$ (namely edges). Such a subsystem is essential when no two of the convex hulls of the $A_{i}^{\prime}$ are parallel. In this case, the cell is a Minkowski sum of 3 edges from the different $A_{i}$ 's which we call a hexagon. Every hexagon can be refined in two possible ways to a regular mixed decomposition and corresponds to an edge of $N(\mathcal{R})$ (see the cubical flips discussed above). A heptagon cell is a Minkowski sum of an $A_{i}$, w.l.o.g. $A_{0}$, and 2 edges $A_{1}^{\prime}, A_{2}^{\prime}$ from $A_{1}, A_{2}$ resp., which form an essential subfamily $\mathcal{A}^{\prime}=$ $\left(A_{0}, A_{1}^{\prime}, A_{2}^{\prime}\right)$. Every heptagon has up to 3 mixed refinements, each of which has a hexagon cell which is the sum of $A_{1}^{\prime}, A_{2}^{\prime}$ and one edge of $A_{0}$, not parallel to any of them. In this general case, a heptagon corresponds to a triangular 2-face of $N(\mathcal{R})$. Two hexagons in $S$ might give rise to the Minkowski sum of two $N(\mathcal{R})$ edges which forms a parallelogram 2-face of $N(\mathcal{R})$, again in case of non-parallelism. An octagon cell is a Minkowski sum of two $A_{i}$, w.l.o.g. $A_{0}, A_{1}$, and an edge $A_{2}^{\prime}$ of $A_{2}$. An octagon corresponds to a $3 \mathrm{~d}$ resultant polytope, thus to a facet of $N(\mathcal{R})$. We call these facets resultant facets (Fig. 1). A heptagon and a hexagon in $S$ correspond to a facet which equals the Minkowski sum of a segment and a triangle, i.e., the sum of a $1 \mathrm{~d}$ and $2 \mathrm{~d}$ resultant polytopes. In generic position, this is a prism facet (Fig. 1). Finally, 3 hexagons in $S$ correspond to a facet which is the Minkowski sum of 3 segments. In generic position this is a cube facet (Fig. 1).

The number of facets. We describe resultant polytopes corresponding to maximum facet cardinality. We start with several lemmata that serve as tools later. Assume we have a hexagon $X=s_{0}+s_{1}+s_{2}$ where $s_{i} \subset A_{i}$ of cardinality 2, and a heptagon $H=A_{0}+s_{1}^{\prime}+s_{2}^{\prime}$ where $s_{i}^{\prime} \subset A_{i}$ is of cardinality 2 for $i \in\{1,2\}$, with the corresponding support sets being essential.

Lemma 5. A heptagon and a hexagon in a subdivision have exactly one common edge from $A_{i}$ for some $i \in\{0,1,2\}$.

Proof. Observe that $X, H$ always share the edge $s_{0} \subset$ $A_{0}$. Then if they have one more common edge, this should be from $A_{1}$ or $A_{2}$. W.l.o.g. it is an edge from $A_{1}$ and we call it $s_{1}$. Then we can construct a subdivision of $A_{0}+s_{1}+A_{2}$ that contains $X, H$. This yields a prism as $3 \mathrm{~d}$ resultant polytope, which cannot exist.

LEMMA 6. There are no two subdivisions which contain the same hexagon and heptagon.

Proof. We will show that by fixing the common edge $s_{0} \subset A_{0}$, there is a unique way to construct a regular mixed subdivision with $X$ and $H$. Assume there are two such subdivisions: one with $X, H$ and one with $X^{*}, H^{*}$, which both share the segment $s_{0}$. We can always subdivide $H$ in a way s.t. it has the hexagon $s_{0}+s_{1}^{\prime}+s_{2}^{\prime}$ as a cell. Similarly, $H^{*}$ has $s_{0}+s_{1}^{*}+s_{2}^{*}$ as a cell, where $s_{i}^{*}, s_{i}^{*} \subset A_{i}$, for $i \in\{1,2\}$. This implies that $s_{0}+A_{1}+A_{2}$ can have two different pairs of hexagons, namely $s_{0}+s_{1}^{\prime}+s_{2}^{\prime}$ and $s_{0}+s_{1}^{*}+s_{2}^{*}$. Thus, the $3 \mathrm{~d}$ resultant polytope of $s_{0}+A_{1}+A_{2}$ has two different parallelogram facets, which is impossible.

COROllary 7. There is no subdivision with an octagon and a hexagon cell or with two heptagon cells.

We now introduce a technical tool. Let $G$ be the dual graph of a regular subdivision $S$ (not necessarily tight); $G$ is planar. The cells, edges and vertices of $S$ correspond, resp., to nodes, edges and cells of $G$. The graph $G$ also contains a special node, which can be placed at infinity, corresponding to the complement of $S$ in the plane. The cells and edges incident to this node are called unbounded. There are 9 unbounded edges incident to this node. The unbounded edges may form multi-edges if at least 2 of them are incident to the same non-infinity node. An example is in Fig. 4, corresponding to the non-regular subdivision $S$ of Fig. 3.

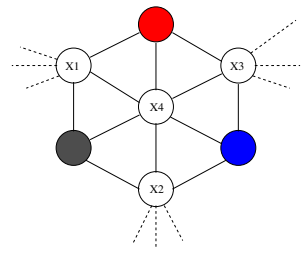

Figure 4: The graph $G$ dual to $S$ of Fig. 3, with labels on the 4 nodes dual to hexagons. Nodes dual to X1, X2, X3 and the triangles (colored) define the perimeter. Unbounded edges are dashed.

Lemma 8. The dual graph $G$ has 9 unbounded cells, each defined by two unbounded edges and up to 2 bounded edges.

Proof. $G$ has as many unbounded cells as unbounded edges, which are as many as the boundary edges of $P$. Each unbounded cell is defined by two unbounded edges and, possibly, some bounded edges. There cannot be $\geq 3$ bounded edges in an unbounded cell because this would imply having $\geq 2$ cells in $S$ with no edge on the boundary $\partial P$. These would have to be triangles sharing an edge, which is impossible if $S$ is obtained by a sufficiently generic lifting.

Unbounded cells with no bounded edges are defined by an unbounded double-edge between the dual of a hexagon cell and the node at infinity. For unbounded cells with 2 bounded edges, the common node between the bounded edges is incident to no unbounded edge hence it is dual to a triangle that intersects $\partial P$ at a vertex; see e.g., the 3 nodes in Fig. 4 dual to triangles in $S$.

Given 3 triangles, no subdivision can have more than 4 hexagons, but the following can occur.

Lemma 9. There exists an essential $(3,3,3)$ family of 2dimensional triangles with no parallel edges, which has a unique subdivision with 4 hexagons. This coarse subdivision is not regular, but it gets refined to regular subdivisions defining different faces of $N(\mathcal{R})$. 
Proof. Existence follows from Example 2 and the subdivision of Fig. 3, with dual graph in Fig. 4. The 4 segments corresponding to the Newton polytopes of the 4 associated resultants of 3 binomials in dimension 2 are linearly independent, and thus cannot define any boundary face of $N(\mathcal{R})$. We detail in Remark 1 its refinements giving rise to faces of $N(\mathcal{R})$.

Lemma 10. For any $\left(A_{0}, A_{1}, A_{2}\right)$ the number of triplets of hexagons $X_{1}, X_{2}, X_{3}$ over all subdivisions of $\sum_{i=0}^{2} A_{i}$ is:

1. at most 1 , if any two $X_{i}$ 's have empty intersection,

2. 0, if more than two pairs of $X_{i}$ 's have a non-empty intersection.

3. 0, if exactly two $X_{i}$ 's have a non-empty intersection,

4. at most 3, if exactly two pairs of $X_{i}$ 's have a non-empty intersection,

Proof. The intersection of $2 X_{i}$ 's is empty or a segment.

Case 1. The hexagons must intersect the boundary: observe that the hexagons use all the edges of $P_{i}$ thus their convex hull should be $P$. Then, there is only one way to put 3 hexagons to intersect the boundary. There is one way to color the edges of the graph in Fig. 4 such that the edges of the hexagons alternate and the edges of the triangles have the same color.

Case 2. If all 3 pairs of $X_{1}, X_{2}, X_{3}$ have non-empty intersection, their convex hull is a 9-gon that contain the 3 hexagons and a triangle in the middle, i.e. it intersects all hexagons but not the boundary of the 9 -gon. The dual graph of this subdivision is a 4-clique. Assume that $A_{i}$ have colors, black, blue, red. Observe that we cannot color the edges of the 4-clique in a way s.t. the edges of the triangle are colored with the same color and no two bounded edges of each hexagons are colored with the same color. The claim follows from the fact that a hexagon is a sum of 3 edges of different colors. Case 3 holds with similar, more complicated arguments.

Case 4. Let $X_{1}=\left\{s_{0}, s_{1}, s_{2}\right\}, X_{2}=\left\{s_{0}^{\prime}, s_{1}, s_{2}^{\prime}\right\}, X_{3}=$ $\left\{s_{0}^{\prime \prime}, s_{1}^{\prime}, s_{2}\right\}$ for segments $s_{i}, s_{i}^{\prime}, s_{i}^{\prime \prime} \subset A_{i}$. The first property is that there is no other triplet of hexagons sharing $s_{1}, s_{2}$, otherwise $A_{0}+s_{1}+A_{2}$ would have another pair of hexagons different than $X_{1}, X_{2}$ which implies that a $3 \mathrm{~d} N(\mathcal{R})$ has 2 parallelogram facets, which is impossible. Thus, if there exists another triplet of hexagons that uses all segments of $A_{0}$, it shares $s_{1}^{\prime} \neq s_{1}$ and $s_{2}^{\prime} \neq s_{2}$. The second property is that the hexagons use all the segments of the triangle $P_{0}$, hence $P_{0}$ should be placed in the middle of them. Thus, each hexagon can form a heptagon with $A_{0}$, namely $A_{0}+s_{1}+s_{2}$, $A_{0}+s_{1}+s_{2}^{\prime}, A_{0}+s_{1}^{\prime}+s_{2}$. Each of these 3 heptagons corresponds to a prism facet. By the first property above we observe that all prism facets produced by different triplets of hexagons are all different. There are at most 9 prism facets by Lem.13, so there are at most 3 cube facets.

Note that the subdivision of Fig. 3 has 4 triplets of hexagons and thus satisfies the statements of Lem. 10 at their extreme.

We are now ready to bound the maximum number of facets.

Lemma 11. If the 3 triangles $A_{i}$ share no parallel edges, then the only possible facets are the ones depicted in Fig 1.
Proof. The uniqueness of the type of the resultant facet comes from Lem. 4. Combining this with Cor. 7 we observe that we cannot have a resultant facet as a Minkowski sum that has a 3-dimensional summand or two 2-dimensional summands. The other possibilities are the Minkowski sum of a segment and a triangle (i.e. a prism facet), or three affinely independent segments (i.e. a cube facet), or four affinely dependent segments. The latter cannot occur since the only subdivision that corresponds to a Minkowski sum of 4 segments gives raise to affinely independent segments in the non parallel case.

We start by bounding the number of resultant facets, they are as the one in Fig. 1(resultant).

LEMMA 12. There can be at most 9 resultant facets in the $(3,3,3)$ case, and this is tight.

Proof. The resultant facet is a $3 \mathrm{~d}$ resultant polytope, corresponding to a subsystem with no parallel edges and support cardinalities $(3,3,2)$. This subsystem, comprised of two triangles and an edge, defines a Minkowski sum equal to an octagon. Consider a coarse mixed subdivision $S$ of $A_{0}, A_{1}, A_{2}$, containing this octagon as a cell. All the other cells of $S$ correspond to non-essential subsystems, hence their resultant is a monomial. There are 9 different subsystems with support cardinalities $3,3,2$, because there are 3 ways to choose the $A_{i}$ contributing an edge, and 3 ways to specify this edge. This bound is tight because it is achieved in Example 2.

The facet in Fig. 1 (resultant) contains 6 vertices, 11 edges, and 7 ridges: 6 triangular and one parallelogram.

A prism facet is the Minkowski sum of a triangular ridge $T$ and an edge $E$ of $N(\mathcal{R})$, see Fig. 1(prism), where $T, E$ are resultant polytopes of subsystems with cardinalities $(3,2,2)$ and $(2,2,2)$ resp. This type of facet has 6 vertices. The ridges are two translates of $T$, and 3 Minkowski sums of $E$ with every edge of $T$. Each prism facet has 9 edges: 3 translates of $E$, and two translates of each edge of $T$. The subdivision of $P$ which corresponds to a prism facet should contain a hexagon $X$ and a heptagon $H$, where $X$ corresponds to $E$ and $H$ to $T$.

Lemma 13. There can be at most 9 prism facets in the $(3,3,3)$ case, and this is tight.

Proof. Consider a hexagon $X=s_{0}+s_{1}+s_{2}$, where $s_{i} \in A_{i}$, and a heptagon $H$, which is the Minkowski sum of $A_{0}$ and segments $s_{1}^{\prime}, s_{2}^{\prime}$, where $s_{i}^{\prime} \subset A_{i}$, for $i \in\{1,2\}$. By Lem. $5, X$ and $H$ should have a common edge from $A_{0}$. By Lem. 6, if we fix $s_{0} \subset A_{0}$, there is a unique way to construct a regular mixed subdivision with $X$ and $H$. Hence, there are at most 9 such subdivisions, which shows there are at most 9 prism facets. This is tight because it is achieved in Example 2.

The cube facet is a Minkowski sum of $3 N(\mathcal{R})$ edges, each corresponding to a hexagon, see Fig. 1(cube). The corresponding (regular) mixed subdivision of $P$ contains 3 hexagons, yielding 3 resultant edges whose Minkowski sum is the cube facet. Each cube facet contains 8 vertices, 12 edges, and 6 parallelogram ridges.

LEMMA 14. There can be at most 4 cube facets in the $(3,3,3)$ case, and this is tight. 

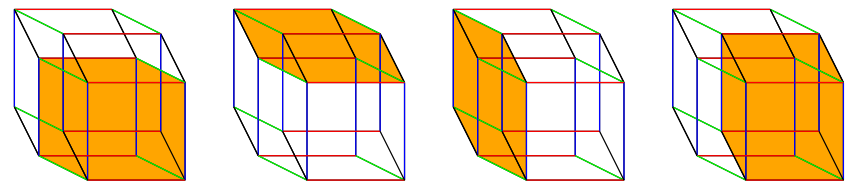

Figure 5: The complex of 4 cube facets: a Minkowski sum of 4 affinely independent segments, each associated to a hexagon in the subdivision; each subfigure highlights a cube.

Proof. In order to have a cube facet we need 3 hexagons in a subdivision. By Lem. 10 the largest number of triplets of hexagons in all subdivisions of a fixed family $\left(A_{0}, A_{1}, A_{2}\right)$ is 4. Thus, there are at most 4 cube facets. The maximum cardinality is achieved by our maximal instance in Ex. 2 where the 4 cube facets are constructed by selecting the 4 different triplets of hexagons from the subdivision of Fig. 3.

REMARK 1. The subdivision of Fig. 3, attained in our maximal instance, corresponds to a Minkowski sum of 4 affinely independent segments, each corresponds to a subdivision's hexagon. As we remarked, this Minkowski sum is 4-dimensional and thus the subdivision could not be regular because it neither corresponds to the whole 4-dimensional polytope nor to any of its faces. We indicate now the topology of the cube facets correspond to refinements of this subdivision. Let $(a, b, c, d) \in\{0,1\}^{4}$ stand for the two possible flips in the 4 hexagons. There are 16 fine subdivisions of $S$ : those which are regular correspond to resultant vertices. Let us denote, w.l.o.g., by

$$
(0 b c d),(a 0 c d),(a b 0 d),(a b c 0) \subset\{0,1\}^{4},
$$

the subsets of regular fine subdivisions defining cubical facets, each with cardinality 8. The flip graph corresponds to 4 cubical facets, each defined by all possible flips in 3 of the hexagons. Hence, each is a neighbor of the other 3 , as shown in Fig. 5, with a parallelogram in common. The facet graph is a 4-clique. Overall, 15 fine subdivisions are involved, hence regular, while one fine subdivision, namely (1111), is non-regular and not contained in any of the 4 facets. Of course, each cube has another 3 parallelograms in common with non-cube facets.

\subsection{The number of faces}

We denote by $\tilde{f}_{i}$ the maximum number of faces of dimension $i$ of any $(3,3,3)$ resultant polytope. It follows from Thm 19 that it is enough to bound the maximal number of faces in the generic case with no parallel edges, considered in Sect. 3.1.

We will make use of a powerful result extending Barnette's Lower bound to non-simplicial polytopes:

Proposition 15. [7, thm.1.4] For d-dimensional polytopes:

$$
f_{1}+\sum_{i \geq 4}(i-3) f_{2}^{i} \geq d f_{0}-\left(\begin{array}{c}
d+1 \\
2
\end{array}\right)
$$

where $f_{2}^{i}$ is the number of 2-faces which are $i$-gons.

The following theorem summarizes our results on the maximum numbers $\tilde{f}_{i}$.
THEOREM 16. . The maximal number of ridges of a $(3,3,3)$ resultant polytope is $\tilde{f}_{2}=66$ and the maximal number of facets is $\tilde{f}_{3}=22$. Moreover, $\tilde{f}_{1}=\tilde{f}_{0}+44,22 \leq \tilde{f}_{0} \leq 28$, and $66 \leq \tilde{f}_{1} \leq 72$. The lower bounds are tight.

Proof. Assume that we have a non parallel $(3,3,3)$ configuration and let us relate $f_{2}$ and $f_{3}$. Let $\phi_{1}, \phi_{2}, \phi_{3}$ be the number of resultant, prism and cube facets resp.; i.e. $\phi_{i}$ is the number of facets with $i$ summands. By Lemma 11, the total number of facets is $f_{3}=\phi_{1}+\phi_{2}+\phi_{3}$. We observe that there are only triangular and parallelogram ridges, whose cardinalities are at most 36 and 30, resp.:

$$
\begin{aligned}
\frac{1}{2}\left(6 \phi_{1}+2 \phi_{2}\right) & =3 \phi_{1}+\phi_{2} \leq 36, \\
\frac{1}{2}\left(\phi_{1}+3 \phi_{2}+6 \phi_{3}\right) & =\frac{1}{2}\left(\phi_{1}+3 \phi_{2}\right)+3 \phi_{3} \leq 30 .
\end{aligned}
$$

The total number of ridges is then

$$
f_{2}=\frac{1}{2}\left(7 \phi_{1}+5 \phi_{2}\right)+3 \phi_{3} \leq 66 .
$$

Thus, $\tilde{f}_{2} \leq 66$ and our maximal instance establishes the lower bound.

With respect to the number of facets, there are at most 9 resultant, 9 prism, and 4 cubical facets by Lemmata 12,13 , and 14. Thus $\tilde{f}_{3} \leq 22$ and again, our maximal instance in Example 2 establishes the lower bound.

By Euler's equality, for any resultant polytope we have $f_{0}+f_{2}=f_{1}+f_{3} \leq \tilde{f}_{1}+\tilde{f}_{3}$, therefore $\tilde{f}_{0}+\tilde{f}_{2} \leq \tilde{f}_{1}+\tilde{f}_{3}$. By symmetry, we get $\tilde{f}_{0}+\tilde{f}_{2}=\tilde{f}_{1}+\tilde{f}_{3}$. Then,

$$
\tilde{f}_{1}-\tilde{f}_{0}=\tilde{f}_{2}-\tilde{f}_{3}=44
$$

With respect to the two last inequalities in the statement, $t$ he lower bounds are given by our maximal instance and by equality (3), it is enough to prove $\tilde{f}_{0} \leq 28$. Again, assume we are in the non parallel case. In the resultant polytope with maximal number of facets, the 2-faces are either triangles or parallelograms and there are $f_{2}^{4}=30$ parallelograms. Prop. 15 becomes $\tilde{f}_{1}+30 \geq 4 \tilde{f}_{0}-10$. Then,

$$
\tilde{f}_{1}+40=\tilde{f}_{0}+84 \geq 4 \tilde{f}_{0},
$$

and the desired bound follows.

\section{CLASSIFICATION}

Let us summarize the characterization of $4 \mathrm{~d}$ resultant polytopes. We need to consider 3 special instances, corresponding to 3 possible cardinalities of supports in Sect. 2.1. As mentioned before, the cases $n=0,1$ are similar to those in [9], so we concentrate on $(3,3,3)$. We fix $n=2$ and $m=9=3+3+3$ and consider such families. The associated mixed Grassmannian $G(2 ; 3,3,3 ; \mathbb{Q})$, defined in $[1]$, is the linear subvariety of the Grassmanian of 5 -dimensional subspaces in $\mathbb{Q}^{9}$ which contain the vectors $e_{1}+e_{2}+e_{3}, e_{4}+e_{5}+e_{6}$, and $e_{7}+e_{8}+e_{9}$. Given a $(3,3,3)$ family $\mathcal{A}$, its associated Cayley matrix $C$ represents (via its rowspan) an element in $G(2 ; 3,3,3 ; \mathbb{Q})$. All $5 \times 9$ matrices representing an element in $G(2 ; 3,3,3 ; \mathbb{Q})$ are affinely equivalent to an integer Cayley matrix of an integer $(3,3,3)$ family and have some structural vanishing minors. In the case of Cayley matrices of essential configurations, not too many minors can be 0 , but there could be parallel vectors and repeated points. In Sturmfels' notation [9], the Newton polytope $\mathcal{N}_{12,111}$ corresponds to two univariate configurations of multisets of 3 points, but 
in the first, two of the points coincide: this is the squarebased pyramid in Fig. 1(b). Thus, this is a degeneration of $\mathcal{N}_{111,111}$, which is the Newton polytope for two univariate configurations with 3 different points each, cf Fig. 1. Note that from the point of view of the Cayley matrix $C$, having a configuration with a repeated point is just an occurrence of the fact that some minors of $C$ vanish, similarly to the existence of parallel edges in $\mathcal{A}$, which is the new feature that we have encountered in the study of 4 -d resultant polytopes.

TheOREM 17. Assume we have an essential family $\mathcal{A}$ of $n+1$ (finite) lattice point configurations in $\mathbb{R}^{n}$ with $N(\mathcal{R})$ of dimension 4. Then, up to reordering, we are in one of the situations (i), (ii) or (iii) in Sect. 2.1. These resultant polytopes are, resp., a degeneration of the following:

1. $n=0,\left|A_{0}\right|=5$, which is a 4-simplex with $f$-vector $(5,10,10,5)$,

2. $n=1,\left|A_{0}\right|=3,\left|A_{1}\right|=4$, which is a Sylvester case, with $f$-vector $(10,26,25,9)$,

3. $n=2,\left|A_{0}\right|=\left|A_{1}\right|=\left|A_{2}\right|=3$, which are the polytopes described in Sect. 3.

In particular, no resultant polytope of dimension 4 can have more than 22 facets and 66 ridges.

Proof. By Thm 3, we restrict our attention to cases 1 to 3 . We discuss case 3 because cases 1 and 2 are settled, resp., in [9] and [5, ch.12], cf also the 8th instance in Table 1.

We can perturb (with values in $\mathbb{Q}$ ), e.g. a point $p \in A_{0}$ to a nearby rational point $p *$. We get a perturbed matrix $C_{\mathbb{Q}}^{\prime} \in G(2 ; 3,3,3 ; \mathbb{Q})$ of the Cayley matrix $C$. The resultant is an affine invariant of a configuration or its Cayley matrix, so we can left multiply $C_{\mathbb{Q}}^{\prime}$ by an invertible matrix $M$ in block form with a $3 \times 3$ identity matrix in the upper left corner and an integer $2 \times 2$ integer matrix $M^{\prime}$ with nonzero determinant in the lower right corner, to get an integer matrix $C^{\prime}=M C_{\mathbb{Q}}^{\prime}$, which corresponds to the same point in the mixed Grassmanian. Then, $C^{\prime}$ is the Cayley matrix of an essential integer family $\mathcal{A}^{\prime}$. We can say that $\mathcal{A}$ is then a degeneration of this new integer family $\mathcal{A}^{\prime}$, which is the image of the family $\mathcal{A}_{\mathbb{Q}}^{\prime}=\left(A_{0}-\{p\} \cup\{p *\}, A_{1}, A_{2}\right)$ by $M^{\prime}$.

Given a regular mixed subdivision $S$ of $\mathcal{A}$ associated to a generic lifting vector $w$ (i.e., $w$ is generic among the vectors that produce the same regular subdivision), we consider the regular subdivision $S_{\mathbb{Q}}^{\prime}$ that $w$ induces on the perturbed configuration $\mathcal{A}_{\mathbb{Q}}$. We then translate $S_{\mathbb{Q}}^{\prime}$ via multiplication by $M^{\prime}$ to a combinatorially equivalent regular subdivision $S^{\prime}$ of $\mathcal{A}^{\prime}$. It follows from Thm 19 that the number of facets of $N(\mathcal{R})$ cannot exceed the number of facets of $N\left(\mathcal{R}^{\prime}\right)$, and we conclude by Thm 16 .

EXAMPLE 4. Let us consider degeneracy when $n=1$, i.e. points are repeated: $A_{0}=\{0,1\}, A_{1}=\{0,1,1,2\}$. We perturb $A_{1}$ and get $A_{1}^{*}=\{0,1,101 / 100,2\}$. We dilate by 100 (multiply a row of the Cayley matrix) and get $B_{0}=$ $\{0,100\}, B_{1}=\{0,100,101,200\}$, which span $\mathbb{Z}$. The resultant polytopes for $\mathcal{A}, \mathcal{B}$ are combinatorially equivalent, although the former resultant has total degree $2+1=3$, and the latter $200+100=300$.

\subsection{Input genericity maximizes complexity}

Given a polytope $Q \subset \mathbb{R}^{3}$ and a direction $u \in \mathbb{R}^{3}$, its lower hull along $u$, denoted $\mathrm{LH}_{u} Q$, is the union of all facets whose outer normal has negative or zero inner product with $u$. In the case of zero inner product, the facet is called degenerate and its projection is not a maximal cell. We assume that the triangles $A_{i}=\left\{p_{i j}, j=0,1,2\right\}, i=0,1,2$, have $2 \mathrm{~d}$ convex hulls $P_{i}$. Let $S$ be a regular subdivision of $P$, and $\widehat{A}_{i}, \widehat{P}$ be the lifted Newton polytopes and their Minkowski sum; $\mathrm{LH}_{e_{3}} \widehat{P}$, where $e_{3}$ is the unit vector on the $x_{3}$-axis, is in bijection with $S$. Consider edges $E_{0}, E_{1}$ with the same outer normal $v$ :

$$
E_{0}=\left(p_{00}, p_{01}\right) \subset P_{0}, E_{1}=\left(p_{10}, p_{11}\right) \subset P_{1} .
$$

For some vertex $p_{2 k} \in A_{2}, E_{1}+E_{2}+p_{2 k}$ is an edge of $P$ with outer normal $v$. Thus, their lifting $\widehat{E}_{1}+\widehat{E}_{2}+\widehat{p}_{2 k}$ has outer normal $(v, 0)$ and yields one or two facets of $\widehat{P}$, yielding one or two degenerate facets on $\mathrm{LH}_{e_{3}} \widehat{P}$, i.e. segments, depending on whether the lifting leads, resp., to a coarse or fine subdivision. In the latter case, the two segments are collinear but their union has been subdivided into one of two possible mixed subdivisions, each with two cells. W.l.o.g., these are:

$\left\{p_{00}+E_{1}+p_{2 k}, E_{0}+p_{11}+p_{2 k}\right\},\left\{E_{0}+p_{10}+p_{2 k}, p_{01}+E_{1}+p_{2 k}\right\}$

We consider a perturbation in the direction of $v$

$$
p_{00}^{*}:=p_{00}+\epsilon v,
$$

with indeterminate $\epsilon \rightarrow 0^{+}$. Since we are considering a finite process that branches on signs of algebraic expressions, namely Cayley minors, $\epsilon$ can take sufficiently small positive rational values, as is the case in standard symbolic perturbation methods.

Lemma 18. With the above hypotheses and notation, let

$$
\mathcal{A}^{*}:=\left(\left\{p_{00}^{*}, p_{01}, p_{02}\right\}, A_{1}, A_{2}\right),
$$

and $P^{*}:=A_{0}^{*}+A_{1}+A_{2}$ be the family and Minkowski sum associated with a perturbation (5). Let $S$ be a (regular fine) mixed subdivision of $P$ associated with a generic weight vector $w$, and $S^{*}$ the regular subdivision of $P^{*}$ associated to the same vector $w$. Then, $S^{*}$ is mixed and contains at most one more cell $\sigma$ than does $S$. There is a bijection between all cells of $S^{*}$ (except $\sigma$, if it exists) and the cells of $S$, which associates combinatorially equivalent cells.

We expect this lemma to extend to any dimension.

Proof. Eq. (5) defines $p_{00}^{*} \in \mathbb{Q}^{2}$. As in the proof of Theorem 17, by an appropriate dilation we define a family of supports in $\mathbb{Z}^{2}$. By abuse of notation, we denote the latter by $\mathcal{A}^{*}$. To prove the lemma for any $S$, we consider two cases according to the subdivisions of $E_{0}+E_{1}+p_{2 k}$ in (4). In the first case, $p_{00}+p_{11}+p_{2 k}$ is not a vertex of $P$ but $p_{00}^{*}+p_{11}+p_{2 k}$ is a vertex of $P^{*}$ : the perturbation has moved outward the middle point of $E_{0}+E_{1}+p_{2 k}$. $\mathrm{LH}_{e_{3}} \widehat{P}$ is combinatorially equivalent to $\mathrm{LH}_{e_{3}+\epsilon v} \widehat{P}$, where the latter is defined by shifting our viewpoint by an infinitesimal amount: the two degenerate facets whose union is $\widehat{E}_{0}+\widehat{E}_{1}+\widehat{p}_{2 k}$ appear in both lower hulls (the subdivision to two facets occurs because $S$ is fine). The non-degenerate facets are clearly combinatorially equivalent in both lower hulls. Formally, non-degenerate facets on $\mathrm{LH}_{e_{3}} \widehat{P}$, i.e. with positive area, have outer normal $(w,-1)$ and we claim that

$$
(w,-1) \cdot\left(e_{3}+\epsilon(v, 0)\right)=-1+\epsilon w \cdot v<0,
$$


for sufficiently small $\epsilon>0$. Thus, these facets also lie on $\mathrm{LH}_{e_{3}+\epsilon v} \widehat{P}$. Non-degenerate facets of $\widehat{P}$ but not on $\mathrm{LH}_{e_{3}} \widehat{P}$ have outer normal $(w, 1)$ and we claim that

$$
(w, 1) \cdot\left(e_{3}+\epsilon(v, 0)\right)=1+\epsilon w \cdot v>0,
$$

for sufficiently small $\epsilon>0$. So, these facets do not lie on $\mathrm{LH}_{e_{3}+\epsilon v} \widehat{P}$. We now show $\mathrm{LH}_{e_{3}} \widehat{P}^{*}$ is combinatorially equivalent to $\mathrm{LH}_{e_{3}+\epsilon v} \widehat{P}$. Any facet except the degenerate ones in $\mathrm{LH}_{e_{3}+\epsilon v} \widehat{P}$ clearly corresponds to a combinatorially equivalent facet in $\mathrm{LH}_{e_{3}} \widehat{P}^{*}$. The degenerate facets give rise to two edges in $\widehat{P}^{*}$, which proves that $S^{*}$ is fine, hence a mixed subdivision; moreover, these edges are combinatorially equivalent to those on $\mathrm{LH}_{e_{3}+\epsilon v} \widehat{P}$. Thus the lemma is proved in the case no new cell is created.

In the second subdivision of $E_{0}+E_{1}+p_{2 k}$, the middle point is $p_{01}+p_{10}+p_{2 k}$; this point is perturbed to the relative interior of $P^{*}$. The perturbation creates an extra (mixed) cell $E_{0}^{*}+E_{1}+p_{2 k}$ which intersects $\partial P^{*}$. For all other cells in $S^{*}$ the discussion for the above case holds. This settles the case a new cell is created.

TheOREM 19. For any family $\mathcal{A}$ whose triangles have one or more pairs of parallel edges, there exists a family of triangles $\mathcal{A}^{*}$ without any parallel edges as in section 3.1, whose resultant polytope $N\left(\mathcal{R}^{*}\right)$ has at least as many faces of any dimension as those in the polytope $N(\mathcal{R})$ of $\mathcal{A}$.

Proof. We first assume all $P_{i}$ 's have non-zero area. Given $\mathcal{A}$ with strongly parallel edges $E_{0} \subset P_{0}, E_{1} \subset P_{1}$, perturbation (5) defines $\mathcal{A}^{*}$, where the corresponding edges are not parallel. In the case of other strongly parallel edges, we apply the same procedure sufficiently many times. For every mixed subdivision $S$ of $\mathcal{A}$ the same lifting defines a mixed subdivision $S^{*}$, as in Lem. 18. This shows that the vertices of $N(\mathcal{R})$ can be mapped in a 1-1 fashion to, possibly a subset of, vertices of $N\left(\mathcal{R}^{*}\right)$. Hence the number of vertices in $N\left(\mathcal{R}^{*}\right)$ is at least as large as that of $N(\mathcal{R})$.

To prove the statement for $k$-faces, $k \geq 1$, we extend Lem. 18 to an arbitrary (coarse) regular subdivision $S$ and its perturbed counterpart $S^{*}$. The only difference is that $S$ may contain a single $1 \mathrm{~d}$ cell $E_{0}+E_{1}+p_{2 k}$ and cells of the form $\sigma=E_{0}+E_{1}+F_{2}$, for a face $F_{2} \subset P_{2}$. Each $\sigma$ is subdivided to 3 or 2 cells in $S^{*}$, depending on whether a new cell is created or not. The subdivision follows one of the subdivisions of $E_{0}+E_{1}+p_{2 k}$ discussed in the proof of Lem. 18. Now $\sigma$ is not essential hence contributes a point summand to the $N(\mathcal{R})$ face corresponding to $S$. The $N\left(\mathcal{R}^{*}\right)$ face corresponding to $S^{*}$ is an edge if $\sigma^{*}$ is a hexagon, thus establishing the lemma for $k$-faces.

If parallel edges $E_{0}, E_{1}$ have antiparallel outer normals, no regular subdivision (even coarse) may contain a cell of the form $E_{0}+E_{1}+F_{2}$, though there may be adjacent cells $E_{0}+p_{1 j}+F_{2}, p_{0 i}+E_{1}+F_{2}$. Any infinitesimal perturbation, such as (5), yields $S^{*}$ combinatorially equivalent to $S$.

When some $P_{i}$ 's have zero area, the result still holds in a similar way after a detailed study of each possible case (including repeated points), which we omit due to space restrictions. The key case is the following: $\mathcal{A}$ satisfies $\left|A_{0}\right|=$ $\left|A_{1}\right|=\left|A_{2}\right|=3, \operatorname{dim} P_{0}=1, \operatorname{dim} P_{1}=\operatorname{dim} P_{2}=2$, then let $\mathcal{A}^{*}=\left(A_{0}^{*}, A_{1}, A_{2}\right)$ such that the middle point of $A_{0}$ is infinitesimally perturbed to yield $\operatorname{dim} P_{0}^{*}=2$. Then there is an injection of regular subdivisions of $\mathcal{A}$ to those of $\mathcal{A}^{*}$, such that if $S$ maps to $S^{*}$ then $S^{*}$ contains one more cell equal to $A_{0}^{*}+p_{1 j}+p_{2 k}$, for vertices $p_{1 j} \in A_{1}, p_{2 k} \in A_{2}$, and all other cells are combinatorially equivalent to the corresponding cells in $S$.

\section{OPEN PROBLEMS AND EXTENSIONS}

Open problem 1. Prove that either $f_{0} \leq 22$ or $f_{1} \leq 66$. That is, we conjecture that the maximum $\bar{f}$-vector of $\bar{a} 4 d$ resultant polytope is $(22,66,66,22)$.

OPEN PROBLEM 2. Is it true that, for maximal $f$-vectors, it holds $f_{0}=f_{3}$ ? Is it always true that $f_{1} \geq f_{2}$, if $f_{0} \geq 10$ ?

The proof of Thm 19 should extend to high dimensions. Lem. 20 generalizes Lem. 12 in any dimension and is proven analogously. It motivates us to raise Conj. 1.

Lemma 20. A d-dimensional resultant polytope has at most $m$ resultant facets.

CONJECTURE 1. The number of vertices of a d-dimensional resultant polytope is bounded above by

$$
\text { 3. } \sum_{\|S\|=d-1} \prod_{i \in S} \tilde{f}_{0}(i)
$$

where $S$ is any multiset with elements in $\{1, \ldots, d-1\}$, $\|S\|:=\sum_{i \in S} i$, and $\tilde{f}_{0}(i)$ is the maximum number of vertices of a $i$-dimensional $N(\mathcal{R})$.

The only bound in terms of $d$ is $(3 d-3)^{2 d^{2}}$ [9], yielding $\tilde{f}_{0}(5) \leq 12^{50}$ whereas our conjecture yields $\tilde{f}_{0}(5) \leq 231$.

\section{Acknowledgments.}

We thank J. de Loera, M. Joswig, F. Santos, and G. Ziegler for helpful discussions and anonymous reviewers for useful comments.

\section{REFERENCES}

[1] E. Cattani, M.-A. Cueto, A. Dickenstein, S. Di Rocco, and B. Sturmfels. Mixed discriminants. Math. Z., 2013. to appear; also in ArXiv 2011.

[2] J. de Loera, J.A. Rambau, and F. Santos. Triangulations: Structures for Algorithms and Applications, vol. 25. Springer-Verlag, 2010.

[3] I.Z. Emiris, V. Fisikopoulos, C. Konaxis, and L. Peñaranda. An output-sensitive algorithm for computing projections of resultant polytopes. In Proc. ACM Symp. Comp. Geometry, pages 179-188, 2012.

[4] I.M. Gelfand, M.M. Kapranov, and A.V. Zelevinsky. Newton polytopes of the classical resultant and discriminant. Advances in Math., 84:237-254, 1990.

[5] I.M. Gelfand, M.M. Kapranov, and A.V. Zelevinsky. Discriminants, Resultants and Multidimensional Determinants. Birkhäuser, Boston, 1994.

[6] A. Jensen and J. Yu. Computing tropical resultants. arXiv:math.AG/1109.2368v1, 2011.

[7] G. Kalai. Rigidity and the lower bound theorem 1. Inventiones Mathematicae, 88:125-151, 1987.

[8] J. Rambau. TOPCOM: Triangulations of point configurations and oriented matroids. In Proc. Intern. Congress Math. Software, pages 330-340, 2002.

[9] B. Sturmfels. On the Newton polytope of the resultant. J. Algebr. Combin., 3:207-236, 1994.

[10] G.M. Ziegler. Lectures on Polytopes. Springer, 1995. 